\title{
STABILITY AND PERFORMANCE ANALYSIS OF A CERTAIN CLASS OF NONLINEAR TIME VARYING FEEDBACK CONTROL SYSTEMS
}

\author{
A. Y. BILAL and L. F. KAZDA* \\ University of Michigan, Ann Arbor, Michigan, U.S.A.
}

\begin{abstract}
Summary-The partitioning technique and the state variable approach have been applied to analyze and to study the behaviour of a control system whose dynamic performance can in general be described by a nonlinear differential equation containing some linear, some nonlinear, and a forcing function terms. By placing suitable restrictions on the system equation, it was found that the state variables, which represent the state of the system, belong to an $L_{2}$ space. Under the assumed restrictions, the admissibie system was found to satisfy the definition of asymptotic stability in the sense of Lyapunov. Utilizing two different definitions of the norm, the system trajectory was analyzed. During the interval of operation considered, expressions for the required norms were obtained inside which all possible states of system remain. Included will be found an example to illustrate the method presented.
\end{abstract}

\section{INTRODUCTION}

THE dynamic behaviour of a broad class of nonlinear physical systems can, in general, be described by an $n^{\text {th }}$ order nonlinear differential equation containing some linear terms, some nonlinear terms and a forcing function. Following the partitioning technique of Wolf [2, 3, 4], the authors analyzed a certain class of these systems [1], and it was found that by placing some restrictions on the system equation, a solution which belongs to an $\mathrm{L}_{2} \dagger$ space was obtained, moreover, this solution was unique.

The objective of this paper is to apply the state variable approach to study and analyze the performance of certain systems under some specific conditions, emphasis being placed upon stability in the sense of LyapunOv $[6,9,10]$, under different definitions of norm $[7,8]$. The authors assume that the reader is familiar with Volterra nonlinear integral equations [5] and has an understanding of LYAPUNOV's theorem of stability.

* The work was supported in part by Project MICHIGAN under Department of the Army Contract DA-36-039 SC-78801, administered by the U.S. Signal Corps and the National Science Foundation.

$\dagger$ See, for example, Appendix A of Reference (1) for a summary of $L_{2}$ space.

[1] A. Y. Bilal and L. F. KAZDA: The Analysis of a Certain Class of Nonlinear Time Varying Differential Equation Systems. Paper submitted to the AIEE Conference, June 1962, and Fublished in Trans. Inst. Elect. Electron. Engrs. Appl. and Indust, May 1963. pp. 78-84.

[2] A. A. Wolf: A Mathematical Theory for the Analysis of a Certain Class of Nonlinear Systems, Doctoral Dissertation, University of Pennsylvania, Philadelphia, Pa. (1958).

[3] A. A. Wolf: Some Recent Advances in the Analysis and Synthesis of Nonlinear Systems, Trans. Amer. Inst. Elect. Engrs. Part II. Appl. \& Industr. (1961).

[4] A. A. Wolf: Generalized Recurrence Relations in the Analysis of Nonlinear Systems, Trans. Amer. Inst. Elect. Engrs. Part I. Commun. \& Electron. (1961).

[5] F. G. Tricomi: Integral Equations, Vol. V. Interscience, New York (1957).

[6] D. R. INGEWERSON: Principal Definitions of Stability, AIEE Workshop on Lyapunov's Second Method, September 1960, published by the University of Michigan, Ann Arbor, Mich., edited by Louis F. Kazda.

[7] Modern Mathematics for Engineers (E. F. BECKENBACH, Ed.), University of California Engineering Extension Series, p. 406. McGraw-Hill, New York (1946).

[8] Coddington and Levinson: Theory of Ordinary Differential Equations, p. 17. McGraw-Hill, New York (1953).

[9] L. Cesari: Problems of Asymptotic Behavior and Stability, Trans. Amer. Inst. Elect. Engrs. Part II. Appl. \& Industr. (1961).

[10] R. E. Kalman and J. E. Betram: Control System Analysis and Design via the Second Method of Lyapunov, J. Basic Engng. (ASME Series D), 371 (1960). 


\section{SYSTEM DESCRIPTION}

The class of system under consideration is described by differential Equation 1 :

$$
L(D, t) x(t)+N\{x\}=g(t)
$$

where $L(D, t)$ is a linear operator, operating on the system output $x(t)$ and is given by:

$$
L(D, t) x(t)=\sum_{m=0}^{n}\left[a_{m}(t) D^{(m)}\right] x(t)
$$

in which the $a$ 's are time-varying parameters, $t$ is the independent variable, and $D^{(m)}=\mathrm{d}^{m} / \mathrm{d} t^{m}$ is the $\mathbf{m}^{\text {th }}$ order differential operator

$N$ is the nonlinear part of the system, which may be a function of $x, \dot{x}, \ldots, x^{n-1}$, and $t$ $g(t)$ is the forcing function.

\section{SYSTEM DEFINITIONS}

(1) $W(t, u)$ is the impulsive response of the linear part of Equation 1, defined by Equation 3:

$$
L(D, t) W(t, u)=\delta(t-u)
$$

where $\delta(t-u)$ is the unit impulse applied at $t=u$.

(2) $x_{0}(t)$ is the response of the linear part of Equation 1 with $g(t)$ as the input forcing function, which for zero initial conditions is defined by Equation 4 :

$$
x_{0}(t)=\int_{0}^{t} W(t, u) g(u) \mathrm{d} u .
$$

(3) The weighted nonlinear function

$$
\begin{aligned}
& F(t, u, x(t)) \triangleq W(t, u) N[x(t)] . \\
& \text { SYSTEM RESTRICTIONS }
\end{aligned}
$$

(1) $\left\{x_{0}^{(m)}(t)\right\}$ belong to an $L_{2}$ space over an interval $[0, T]$ and are assumed bounded $m=0,1, \ldots,(n-1)$, where $\mathrm{n}$ is the order of the highest derivative of the linear part of the system equation. $(m)=$ the $m^{\text {th }}$ order derivative with respect to the independent variable $t$.

(2) In a given region $D ;[|x| \leqslant d(t), 0 \leqslant u \leqslant t \leqslant], F^{(m)}[t, u, x(u)]^{*}$ satisfies the following two conditions:

(a) $F^{(m)}[t, u, x(u)]$ satisfies the modified Lipschitz condition, namely, if the two triplets $\left(t, u, z_{1}\right)$ and $\left(t, u, z_{2}\right)$ are in $D$, then

$$
\left|F^{(m)}\left(t, u, z_{1}\right)-F^{(m)}\left(t, u, z_{2}\right)\right| \leqslant K_{m}(t, u)\left|z_{1}-z_{2}\right|
$$

where $\left[K_{m}(t, u)\right]$ are $L_{2}[0, T]$. That is,

(b) $\int_{0}^{T} F^{(m)}\left[t, u, x_{0}(u)\right] \mathrm{d} u \leqslant n_{m}(t)$

$$
\int_{0}^{t} K_{m}^{2}(t, u) \mathrm{d} u \leqslant \alpha_{m}^{2}(t), \quad \int_{0}^{T} \alpha_{m}^{2}(t) \mathrm{d} t \leqslant A_{m}^{2} .
$$

${ }^{*} F(m)(t, u, x) \triangleq \frac{\partial(m) F(t, u, x)}{\partial t^{m}}$ and $F$ is considered as a function of $x$ only and not $x$, etc. 
where $n_{m}(t)$ are bounded and belong to an $L_{2}[0, T]$, that is,

$$
\int_{0}^{T} n_{m}^{2}(t) \mathrm{d} t \leqslant C_{m}^{2}
$$

where $m$ and $(m)$ are as defined before

$A_{m}^{2}$ and $C_{m}^{2}$ are finite positive numbers

$T$ is the period of interest, $(0<T \leqslant \infty)$.

The functions $\alpha_{m}^{2}(t)$ and $n_{m}(t)$ are continuous and bounded over $[0, T]$.

(3) It is required that:

$$
\left|x_{0}(t)\right|+|n(t)|+C \alpha(t) \sum_{k=0}^{\infty} \frac{A^{k}}{\sqrt{k !}} \leqslant d(t) .
$$

(4) The time-varying parameters $\alpha_{i}(t)$ and $|N(x, t)-g(t)|$ are bounded over $[0, T]$.

It should be noted that when $m=0$, the set of restrictions 6 through 10 reduces to the set of restrictions already described by the authors in Reference [1] where it was proved, that for the system described by Equation 1 for the case where there exists a unique solution $x(t)$ which is the limit of a sequence of iterates $\left\{x_{n}(t)\right\}$, and which, together with the system solution $x(t)$, belongs to an $L_{2}$ space. This sequence of iterates $\left\{x_{n}\right\}$ is given by Equation 11 :

$$
x_{n+1}(t)=x_{0}(t)-\int_{0}^{t} F\left[t, u, x_{n}(u)\right] \mathrm{d} u, \quad(n=0,1,2, \ldots, \infty) .
$$

\section{THE STATE VARIABLE REPRESENTATION}

Consider the system described by Equation 1 and satisfying the restrictions given by Expressions 6 through 10. Let

$$
\left.\begin{array}{c}
x=v_{1} \\
\frac{\mathrm{d} x}{\mathrm{~d} t}=v_{2} \\
\vdots \\
\frac{\mathrm{d} x^{n-1}}{\mathrm{~d} t}=v_{n}
\end{array}\right\}
$$

Then:

$$
\left.\begin{array}{lc}
\frac{\mathrm{d} v_{1}}{\mathrm{~d} t}=v_{2} & =f_{1}\left(t, v_{1}, v_{2}, \ldots, v_{n}\right) \\
\frac{\mathrm{d} v_{2}}{\mathrm{~d} t}=v_{3} & =f_{2}\left(t, v_{2}, v_{3}, \ldots, v_{n}\right) \\
\vdots & \vdots \\
\frac{\mathrm{d} v_{n}}{\mathrm{~d} t}=-a_{0}(t) v_{1}-a_{1}(t) v_{2}, \ldots+\left(N_{1}\right) & =f_{n}\left(t, v_{1}, v_{2}, v_{3}, \ldots, v_{n}\right)
\end{array}\right\}
$$


where

$$
N_{1}=N_{1}(x, t)=g(t)-N(x, t)
$$

and $N$ and $g$ are as defined above. Equation $I$ can be put into matrix notation:

$$
\frac{\mathrm{d}}{\mathrm{d} t}\left[\begin{array}{c}
v_{1} \\
v_{2} \\
\vdots \\
v_{n}
\end{array}\right]=\left[\begin{array}{cccccc}
0 & 1 & 0 & \ldots & 0 & 0 \\
0 & 0 & 1 & \ldots & 0 & 0 \\
0 & 0 & 0 & \ldots & 0 & 1 \\
-a_{0}(t) & -a_{1}(t) & -a_{2}(t) & \ldots & -a_{n-2}(t) & -a_{n-1}(t)
\end{array}\right]\left[\begin{array}{c}
v_{1} \\
v_{2} \\
\vdots \\
v_{n}
\end{array}\right]+\left[\begin{array}{c}
0 \\
0 \\
\vdots \\
N_{1}
\end{array}\right](14)
$$

Equation 14 can also be represented by Equations 15 :

and

$$
\left.\begin{array}{l}
\left.\left.\left.\frac{\mathrm{d}}{\mathrm{d} t} v\right]=[A(t)] v\right]+N_{1}\right] \\
\frac{\mathrm{d}}{\mathrm{d} t} \mathbf{v}=\mathbf{f}(t, \mathbf{v})
\end{array}\right\}
$$

where $\mathrm{v}$ is a vector composed of the state variables $\left(v_{1}, v_{2}, \ldots, v_{n}\right)$ and can be used to represent the state of the system in the phase space. Each $v_{i}$ represents an axis in the phase space in which each succeeding axis represents the rate of change of the quantity measured along the one preceding it.

$\mathbf{f}$ is a column vector made of the functions $\left(f_{1}, f_{2}, \ldots, f_{n}\right)$ as defined by Equation 13 . $[A(t)]$ is an nxn matrix, which is defined by Equation (14).

\section{THE PROPERTIES OF THE STATE VARIABLES OF THE CLASS OF SYSTEMS UNDER CONSIDERATION}

Some of the properties of the state variables $v_{1}, v_{2}, \ldots, v_{n}$ which are of interest in this study can be summarized in the following theorem:

Theorem:

Under the restrictions (given by Equations 6 through 10) placed on the linear, nonlinear, and forcing function terms, the state variables $v_{i}(t)$ belong to $L_{2}[0, T]$, and are bounded by corresponding arbitrary $L_{2}[0, T]$ functions $\beta_{i}(t)$. Since $\left|N_{1}(t, v)\right|$ and $\left|x_{i}(t)\right|$, which were defined before, are assumed bounded over the interval $[0, T]$, then the state variables $v_{i}(t)$ are uniformly continuous over the same interval $[0, T]$. In particular, if $T=+\infty$, then it follows that

Proof:

$$
\operatorname{Lim}_{t \rightarrow x_{i}} v_{i}(t)=0, \quad(i=1,2, \ldots, n)
$$

The following two lemma are needed to prove the theorem:

\section{Lemma I $^{*}$}

If a function $f(t)$ has a bounded derivative on any interval (finite or infinite); then $f(t)$ is uniformly continuous over the same interval.

* Lemma I can be obtained by applying the law of the mean, namely:

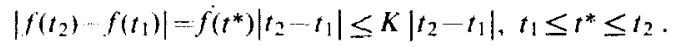




\section{Lemma $I^{*}$}

If a function $f(t)$ is uniformly continuous over the interval $[0, \infty)$ and if

$$
\int_{0}^{\infty} f^{2}(t) \mathrm{d} t
$$

is finite, then it follows that

$$
\operatorname{Lim}_{t \rightarrow \infty} f(t)=0
$$

Recalling that the state variable $v_{1}=x(t)$ is given by the limit as $\mathrm{n}$ tends to infinity of $x_{n}(t)$ in Equation 11, restated again here. namely:

$$
x(t)=v_{1}=\lim _{n \rightarrow \infty} x_{n}(t)=x_{0}(t)-\int_{0}^{t} F\left[t, u, v_{1}(u)\right] \mathrm{d} u \dagger
$$

and that,

$$
\frac{\mathrm{d}}{\mathrm{d} t} \int_{0}^{t} g(t, u) \mathrm{d} u=g(t, t)+\int_{0}^{t} \frac{\mathrm{d}}{\mathrm{d} t} g(t, u) \mathrm{d} u
$$

which gives upon differentiation of Equation 16 once, the following equation:

$$
\frac{\mathrm{d} x}{\mathrm{~d} t}=\frac{\mathrm{d} v_{1}}{\mathrm{~d} t}=\frac{\mathrm{d}}{\mathrm{d} t}\left[x_{0}(t)\right]-F\left[t, t, v_{1}(t)\right]-\int_{0}^{t} \frac{\mathrm{d}}{\mathrm{d} t} F\left[t, u, v_{1}(u)\right] \mathrm{d} u
$$

but since from definition (5)

$$
F\left[t, u, v_{1}(t)\right]=W(t, u) N\left[v_{1}(t)\right]
$$

we can conclude that $F\left[t, u, v_{1}(t)\right]=W(t, t) N\left[v_{1}(t)\right]=0$, since $W(t, t)=0$. Equation 18 can now be put in the form of Equation 19:

$$
x(t)^{(1)}=v_{2}=v_{1}^{(1)}=x_{0}^{(1)}(t)-\int_{0}^{t} F^{(1)}\left[t, u, v_{1}(u)\right] \mathrm{d} u
$$

where as defined before, the superscript (1) denotes the differential operator $\mathrm{d} / \mathrm{dt}$, and

$$
F^{(1)}\left[t, u, v_{1}(u)\right]=W^{(1)}(t, u) N_{1}\left[\dot{v}_{1}(u)\right] .
$$

Recail also that $W(t, t)=W^{(1)}(t, t)=\ldots=W^{(n-2)}(t, t)=0$; therefore, $W^{(n-1)}(t, t)=1 / a_{n}(t) \neq 0$. Differentiating Equation $16 k$ times gives

$$
x^{(k)}=v_{k+1}=x_{0}^{(k)}(t)-\int_{0}^{t} F^{(k)}\left[t, u, v_{1}(u)\right] \mathrm{d} u
$$

where $k=0,1,2, \ldots, n-1$.

* See Ref. 11, p. 86.

†See Ref. 1, Equation 12.

[11] A. C. ZAANEN: Linear Analysis, p. 86. North Holland, Amsterdam (1956). 
Equation 20 gives all the state variables $v_{1}, v_{2}, \ldots, v_{n}$; and for the case when $k=0$, it yields the state variable $v_{1}$, which was previously shown to be an $L_{2}$ function.*

Now to prove that all the $v_{i},(i=1,2, \ldots, n)$ belong to an $L_{2}$ space, we define a sequence $x_{m}^{(k)}$ according to the recurrence relation of Equation 21 :

$$
x_{m+1}^{(k)}=x_{0}^{(k)}(t)-\int_{0}^{t} F^{(k)}\left[t, u, x_{m}(u)\right] \mathrm{d} u
$$

where $m=0,1, \ldots, \infty$ and $k=0,1,2, \ldots, n-1$. Therefore,

$$
x_{m}^{(k)}=x_{0}^{(k)}(t)-\int_{0}^{t} F^{(k)}\left[t, u, x_{m-1}(u)\right] \mathrm{d} u .
$$

Subtracting Equation 21 from 22, and squaring the result gives:

$$
\begin{aligned}
x_{m+1}^{(k)}-x_{m}^{(k)}= & \left(\int_{0}^{t}\left\{F^{(k)}\left[t, u, x_{m}(u)\right]-F^{(k)}\left[t, u, x_{m-1}(u)\right]\right\} \mathrm{d} u\right)^{2} \\
& \leqslant\left\{\int_{0}^{t}\left[K_{k}(t, u)\left|x_{m}(u)-x_{m-1}(u)\right|\right] \mathrm{d} u\right\}^{2} \\
& \leqslant\left\{\int_{0}^{t} K_{k}(t, u)^{2} \mathrm{~d} u \int_{0}^{t}\left[x_{m}(u)-x_{m-1}(u)\right]^{2} \mathrm{~d} u\right\}
\end{aligned}
$$

Therefore

$$
\left[x_{m+1}^{(k)}-x_{m}^{(k)}\right]^{2} \leqslant \alpha_{k}^{2}(t) \int_{0}^{t}\left(x_{m}-x_{m-1}\right)^{2} \mathrm{~d} u \dagger .
$$

Recall that

$$
\int_{0}^{T}\left[x_{m}(u)-x_{m-1}(u)\right]^{2} \mathrm{~d} u \leqslant \frac{C_{0}^{2} A_{0}^{2 m-2 \ddagger} \tau}{(m-2) !} .
$$

Substitution of Equation 25 into Equation 24 gives

$$
\left[x_{m+1}^{(k)}(t)-x_{m}^{(k)}(t)\right]^{2} \leqslant \frac{C_{0}^{2} A_{0}^{2 m-2}}{(m-2) !} \cdot \alpha_{k}^{2}(t)
$$

where $m=2.3 . \ldots, \infty$ and where $k=0,1, \ldots, n-1 ; n$ is the order of the system. It follows therefore that all $x_{m}^{(k)}$ are $L_{2}$ functions.

It can be shown that $\S$

$$
\operatorname{Lim}_{m \rightarrow \infty} x_{m}^{(k)}=x^{(k)}=v_{k+1}
$$

and therefore all the state variables $v_{i}(t)(i=1,2, \ldots, n)$ belong to an $L_{2}$ space in the assumed interval of interest $T$. Using Equation 74 of Appendix $A$, an upper-bound function for each of the state variables $v_{i}(t)$ can be calculated such that:

$$
\left|v_{i}(t)\right| \leqslant \beta_{i}(t) \text { for } i=1,2, \ldots, n, \quad 0 \leqslant t \leqslant T .
$$

* See Ref. 1, Theorems 1, 2, and 3, where it is shown that $v_{1}$ exists and is unique.

${ }^{2}$ Notice that $\int_{0}^{t}<\int_{0}^{T}$ since $t<T$ and the integrand is a positive quantity.

$\ddagger$ See Ref. 1 .

\$See Appendix A for proof of Equation 27. 
The functions $\beta_{i}(t)$ which satisfy Inequality 28 are given by

$$
\beta_{i}(t)=\left|x_{0}^{(i-1)}(t)\right|+\left|n_{i-1}(t)\right|+m\left|\alpha_{i-1}(t)\right|
$$

where $x_{0}^{(i-1)}(t), n_{i-1}(t)$ and $\alpha_{i-1}(t)$ are functions defined by Equations $4,6,7$, and 8 . $m$ is a positive constant given by

$$
m=C_{0}\left(1+A_{0} \sum_{k=0}^{\infty} \frac{A_{0} k}{k !}\right) .
$$

It is clear from Equation 29 that the functions $\left\{\beta_{i}(t)\right\}$ are in $L_{2}$ space, since the righthand side of this equation is a linear combination of functions that are in the $L_{2}$ space. The upper-bound functions $\beta_{i}(t)$ can be changed at will, since they are dependent only on known functions, as defined by the system restrictions, and hence by the system parameters.

If the period of interest $T$ is chosen to be infinite, then from the properties of the $L_{2}$ spaces we can conclude that:

$$
\int_{0}^{\infty} v_{i}^{2}(t) \mathrm{d} t<\infty \text { for } i=1,2, \ldots, n .
$$

It is not necessary for a continuous function, which is square integrable on the interval $[0,+\infty)$, to have a zero limit at $t=+\infty$. However, as shown in Appendix B, the limit may not exist. Utilizing the matrix Equation 14, it can be shown that since the time-varying parameters $a_{n}(t)$ and the nonlinear function $N_{1}(t)$ are assumed bounded, each of the state variables $v_{i}(t)$ has a bounded derivative in the interval of interest (assumed infinite). Thus Lemma I shows that $v_{i}(t)$ for $i=1,2, \ldots, n$ are uniformly continuous over the same interval. Now by considering Equation 31 and by applying Lemma II, it can be concluded that

$$
\operatorname{Lim}_{t \rightarrow \infty} v_{i}(t)=x \text { for } i=1,2, \ldots, n \text {. }
$$

\section{STABILITY CONSIDERATIONS}

In the system described by the vector equation (15), an equilibrium position $\mathbf{v}_{e}$ exists if the state variables have values $v_{1 e}, v_{2 e}, \ldots, v_{n e}$ such that all the derivatives $\mathrm{d} v_{i} / \mathrm{d} t$, where $\mathrm{i}=1,2, \ldots, n$, are simultaneously zero. It can be easily seen from Equation 11 that for the system under consideration the origin of the phase space $\mathbf{v}=\left(v_{1}, v_{2}, \ldots, v_{u}\right)=0$ is a position of equilibrium, under the condition that:

$$
N_{1}(\mathbf{v}, t)=0 \text { at } \mathbf{v}(t)=\mathbf{0} \text {. }
$$

Thus, under the condition given in Equation 32, the solution $\mathbf{v}=\mathbf{0}$ is an equilibrium position of Equation 15 and its stability is in question. With the passage of time, the state vector $\mathbf{v}$, whose components $v_{1}, v_{2}, \ldots, v_{n}$, traces a curve in the phase space known as the system trajectory which describes all possible states of the system. A simple measure of the departure of one possible state $\mathbf{v}$ from the equilibrium position $\mathbf{v}=\mathbf{0}$ can be obtained from the norm of $\mathbf{v}$ denoted by $\|\mathbf{v}\|$ such that:*

(1) $\|\mathbf{v}\|$ is defined for every $\mathbf{v}$ in the space

(2) $\|\mathbf{v}\|=\mathbf{0}$ if and only $\mathbf{v}=0$

(3) $v>0$ for all $v>0$

(4) $\mathbf{v}+\mathbf{u}\|\leq\| \mathbf{v}\|+\| \mathbf{u} \|$ for all $\mathbf{v}$ and $\mathbf{u}$

(5) $\|\alpha \mathbf{v}\|=|\alpha| \cdot\|\mathbf{v}\|$ for all $\mathbf{v}$ and $\alpha$ is a real constant

* See Ref. 7, p. 406. 
Some commonly used norms are:*

$$
\begin{gathered}
\|\mathbf{v}\|=\left[\sum_{i=1}^{n}\left(\mathbf{v}_{i}^{2}\right)\right]^{\frac{1}{2}} \\
\|\mathbf{v}\|=\max _{i}\left\{\left|v_{i}\right|\right\} \\
\|\mathbf{v}\|=\sum_{i=1}^{n}\left|v_{i}\right| \\
\|\mathbf{v}\|=\left[\int_{R} \int_{R}\left|v\left(s_{1}, \ldots, s_{v}\right)\right|^{2} \mathrm{~d} s_{1}, \ldots, \mathrm{d} s_{v^{\prime}}\right]^{\frac{1}{2}}
\end{gathered}
$$

where $R$ is a fixed region of the $s_{1}, \ldots, s_{v}$ space, and $|\mathbf{v}|$ denotes the modulus of the vector $\mathbf{v}$ in the usual way. It can be readily shown that the norms as defined by Equations 34 through 37 satisfy the conditions for the norm as stated by Equation 33. Thus we can now say that, for the system under consideration, an output state $\mathbf{u}$ is greater than an output state $\mathbf{v}$ if and only if $\|\mathbf{u}\|>\|\mathbf{v}\|$. From the engineering point of view, we would like a system to have the following property: For all bounded inputs, all possible output states $\mathbf{v}(t)$ must remain below an arbitrary upper-bound state $\mathbf{C}$ for $0 \leq t \leq \infty$, as shown in Fig. 1. This means that the system under consideration is stable. However, in order to investigate stability it is necessary to define precisely the concepts of stability that should be applied.

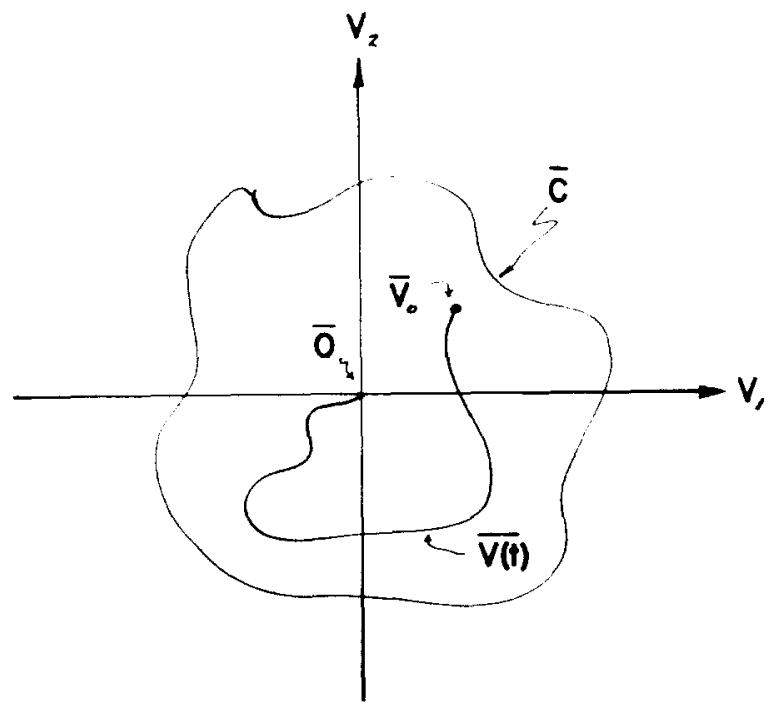

FIG. 1. Definition of stability.

$\mathbf{c}=$ an upper-bound state:

$\mathbf{v}=$ system trajectory;

$v_{0}=$ initial state;

$\mathbf{0}=$ :equilibrium state.

* See Ref. 8, p. 17, for the relation of the different norms. 


\section{DEFINITION OF STABILITY*}

The equilibrium position $\mathbf{v}=\mathbf{0}$ is called stable in the sense of LYAPUNOV if for every $\varepsilon>0$ and for all $t>0$ there exists a $\delta(\varepsilon)>0$ such $\|\mathbf{v}(t)\|<\varepsilon$ whenever $\|\mathbf{v}(0)\|<\delta$. In other words, the equilibrium position $\mathbf{v}=\mathbf{0}$ is stable if the magnitude of the state vector $\mathbf{v}$ can be made to remain below an arbitrary upper bound, when the initial magnitude chosen for it is sufficiently small. If $\mathrm{v}(t)$ approaches zero as $t$ approaches infinity, that is, $\operatorname{Lim}_{t \rightarrow \infty} v_{i}(t)=0$ $(i=1,2, \ldots, n)$, the equilibrium pcsition in this sense is called asymptotically stable.

In applying this definition of stability we have to confine ourselves to a particular definition of the norm $\|\mathbf{v}\|$. However, we are interested in studying the behaviour of the system under the two definitions of norm, as given by Equations 36 and 37, for the following reasons:

(1) The norm as defined by Equation 36 simply gives the magnitude or length of the state vector $\mathbf{v}(t)$, at any particular time $t$, from the equilibrium position $\mathbf{v}=\mathbf{0}$.

(2) The norm as defined by Equation 37 gives a measure of the root mean square value of the state vector $\mathbf{v}(t)$ during the period of interest.

Hence the two definitions of the norm $\|\mathbf{v}\|$ given above govern the behaviour of the system under consideration at any particular time $t$; this is frequently of interest to many systems engineers. Now, for any initial state $v_{0}$, if conditions given by expressions 6 through 10 of the restrictions are true, we can conclude that:

(a) The system under consideration possesses a unique solution.

(b) The system trajectory $\mathbf{v}(t)=\left(v_{1}, v_{2}, \ldots, v_{n}\right)$ approaches $\mathbf{0}$ as the time $t$ approaches infinity,

(c) The norm $\|\mathbf{v}\|$ as defined by Equation 34,

$$
\|\mathbf{v}\|_{\|}=\left[\sum_{i=1}^{n}\left(v_{i}^{2}\right)\right]^{\frac{1}{2}}
$$

is bounded by some arbitrary upper norm $\|\mathbf{B}(t)\|$ given by

$$
\|\mathbf{B}(t)\|=\left[\sum_{i=1}^{n}\left[\beta_{i}^{2}(t)\right]\right]^{\frac{1}{2}}
$$

where $\beta_{i}(t)$ is as previously defined by Equation 29 .

(d) The norm $\|v\|$ as defined by Equation 37,

$$
\|\mathbf{v}\| \triangleq\left[\int_{0}^{\infty}|\mathbf{v}(t)|^{2} \mathrm{~d} t\right]^{\frac{1}{2}} \dagger
$$

exists and is less than an arbitrary positive number $N$, given by Equation 39:

$$
N^{2}=\int_{0}^{\infty}|\mathbf{B}(t)|^{2} \mathrm{~d} t
$$

where

$$
|\mathbf{B}(t)|=\left[\sum_{i=1}^{n} \beta_{i}^{2}(t)\right]^{\frac{1}{2}} .
$$

* See Refs. 6, 9, and 10.

$\dagger$ This equation is a special case of the general equation 37 ; here $v=1, s=t$, and $R$ is the interval $0 \leq t \leq \infty$. 
One can easily see that the two integrals given by Equations 37 and 39 have finite values since both the vector functions $\mathbf{v}(t)=\left(v_{1}, v_{2}, \ldots, v_{n}\right)$ and $\mathbf{B}(t)=\left(\beta_{1}, \beta_{2}, \ldots, \beta_{n}\right)$ are $L_{2}$ functions, as previously proved. It should be noticed however, that the upper-bound norms $\|\mathbf{B}(t)\|$ and $N^{2}$ of Equations 38 and 39 , respectively, are functions of the initial state $\mathbf{v}_{0}(t)=$ $\left(v_{10}, v_{20}, \ldots, v_{n 0}\right)$; therefore we can conclude that if the system starts with an initial state $\mathbf{v}_{0}(t)$ such that $\left\|v_{0}(t)\right\| \leq \delta$ for any given number $\delta$, we can find a number $\varepsilon$ such that $\| \bar{\nu}(t) \mid<\varepsilon$ for $t>t_{1}$, and which depends on $\delta$. Consequently the system under consideration is stable according to the stability definition above.

\section{ILLUSTRATIVE PROBLEM}

The above techniques will now be utilized to analyze the second-order nonlinear control system shown in Fig. 2. The equation of motion for this system is

$$
J \ddot{c}(t)+\left(B+K_{a} K_{b}\right) \dot{c}(t)+K_{a} K_{c} c(t)=K_{a} K_{2 N}(t) N\left(v_{1}\right)
$$

where $v_{1}=[r(t)-c(t)]$ is the actuating error signal with $r(t)=$ the reference input

$c(t)=$ the controlled variable

$B=$ the viscous friction

$N\left(v_{1}\right)=$ a nonlinear element

$K_{2 N}(t)=$ a deterministic time varying input disturbance

$K_{a}, K_{b}, C_{c}$, are constant gains

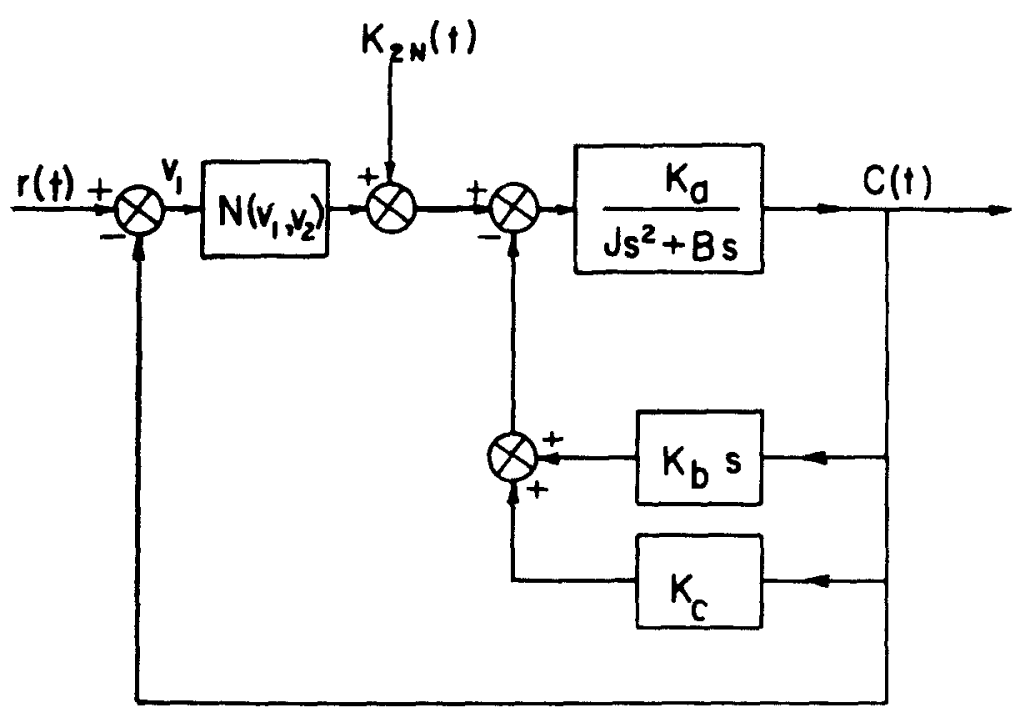

Fig. 2. A second-order nonlinear servo. 
Substituting Equations 42,

$$
\left.\begin{array}{l}
v_{1}=r(t)-c(t) \\
\dot{v}_{1}=\dot{r}(t)-\dot{c}(t) \\
\ddot{v}_{1}=\ddot{r}(t)-\ddot{c}(t)
\end{array}\right\}
$$

into Equation 41 gives:

$$
J \ddot{v}_{1}(t)+\left(B+K_{a} K_{b}\right) \dot{v}_{1}(l)+K_{a} K_{c} \dot{v}_{1}(t)+K_{a} K_{2}(t) N\left(v_{1}\right)=J \ddot{r}(t)+\left(\dot{B}+K_{a} K_{b}\right) \dot{r}+K_{a} K_{c} r
$$

For $r=0$, Equation 43 assumes the following form:

$$
J \ddot{v}_{1}(t)+\left(B+K_{a} K_{b}\right) \dot{v}_{1}(t)+K_{a} K_{c} v_{1}(t)+K_{a} K_{2 N}(t) N\left(v_{1}, v_{2}\right)=0 \text {. }
$$

Equation 44 can be put into the following form:

$$
\left.\begin{array}{lr}
\frac{\mathrm{d} v_{1}}{\mathrm{~d} t}=0+K_{12} v_{2} & =f_{1}\left(v_{1}, v_{2}, t\right) \\
\frac{\mathrm{d} v_{2}}{\mathrm{~d} t}=K_{21} v_{1}+K_{22} v_{2}+K_{2 N} N\left(v_{1}, v_{2}\right) & =f_{2}\left(v_{1}, v_{2}, t\right)
\end{array}\right\}
$$

with $K_{12}, K_{21}$, and $K_{22}$ as given by Equation 46 :

$$
\left.\begin{array}{l}
K_{22}=\frac{-\left[B+K_{a} K_{b}\right]}{J} \\
K_{12} K_{21}=\frac{-K_{a} K_{c}}{J} \\
K_{12}=-\frac{K_{a}}{J}
\end{array}\right\}
$$

assuming the functions $N\left(v_{1}, v_{2}\right)$ and $K_{2 N}(t)$ to be

$$
\left.\begin{array}{l}
N\left(v_{1}, v_{2}\right)=v_{1}^{2} \\
K_{2 N}(t)=e^{2 a t}
\end{array}\right\}
$$

where $\alpha$ is an arbitrary constant that must be determined.

Now, the problem at hand is: Given a nonlinear function $N\left(v_{1}, v_{2}\right)$ and the specific form of $K_{2 N}(t)$ for the control system shown in Fig. 2, choose the parameters $K_{12}, K_{21}$, $K_{22}$ and $\alpha$ so that:

(1) The actuating error signal $v_{1}$ and the rate of change of this signal, $\dot{v}_{1}$, belong to an $L_{2}$ space in the interval $0 \leq t \leq \infty$.

(2) Given two specific numbers $H_{1}=12$ and $H_{2}=32$ for the integrated squared errors, the system satisfies the following:
(a) $\left.\int_{0}^{\infty} v_{1}^{2}(t) \mathrm{d}(t) \leqslant 12\right\}$
(b) $\left.\int_{0}^{\infty} \dot{v}_{1}^{2}(t) \mathrm{d} t \leqslant 32,\right\}$ 
This means that the unknown parameters are required to keep the integrated squared error $\left[v_{1}(t)\right]$ and the integrated squared rate of change of the error $\left[\dot{v}_{1}(t)\right]$ below certain specified levels. Equation 48, also requires that the state variables $v_{1}$ and $v_{2}=i_{1}$ should reach the equilibrium position at the origin of the phase plane as $t \rightarrow \infty$.

To solve this problem it is sufficient to choose the required parameters so that the system under consideration satisfies the restrictions defined by Equations 6 through 10 . In the given domain, Restriction 9 should be checked, otherwise the technique presented here cannot be applied. This means that the technique presented is more flexible when the system has parameters to be chosen.

In order to solve for $x_{0}(t)$, we consider the following linear equations:

$$
\ddot{v}_{1}(t)+\left(\frac{B+K_{a} K_{b}}{J}\right) \dot{v}_{1}(t)+\left(\frac{K_{a} K_{c}}{J}\right) v_{1}(t)=0
$$

with $x_{0}=1, \dot{x}_{0}=0$ at $t=0$. Therefore,

$$
\begin{aligned}
& x_{0}(t)=\frac{1}{m_{1}-m_{2}}\left(-m_{2} e^{m_{1} t}+m_{1} e^{m_{2} t}\right) \\
& x_{0}^{(1)}(t)=\frac{1}{m_{1}-m_{2}}\left(-m_{1} m_{2} e^{m_{1} t}+m_{1} m_{2}\right)
\end{aligned}
$$

where $m_{1}$ and $m_{2}$ are given by

$$
m_{i}=-\left(\frac{B+K_{a} K_{b}}{2 J}\right) \pm \sqrt{\frac{\left(B+K_{a} K_{b}\right)^{2}}{4 J^{2}}-\frac{K_{a} K_{c}}{J}} \quad i=1 \text { and } 2
$$

Also, the impulse response $W(t, u)$ of Equation 49 is given by

$$
\left.\begin{array}{rlrl}
W(t, u)=W(t-u) & =\frac{1}{m_{1}-m_{2}}\left[e^{m_{1}(t-u)}-e^{m_{2}(t-u)}\right] & & t>u \\
& =0 & & t<u
\end{array}\right\}
$$

Using Equation 5 gives

$$
\begin{gathered}
F\left(t, u, v_{1}\right)=\frac{m_{3}}{m_{1}-m_{2}}\left[e^{m_{1}(t-u)}-e^{m_{2}(t-u)}\right] e^{2 \alpha u} v_{1}^{2} \\
F^{(1)}\left(t, u, v_{1}\right)=\frac{m_{3}}{m_{2}-m_{2}}\left[m_{1} e^{m_{1}(t-u)}-m_{2} e^{m_{2}(t-u)}\right] e^{2 \alpha u} v_{1}^{2}
\end{gathered}
$$

where $m_{3}=K_{a} / J$.

For a domain $D=[|x| \leq 1,0 \leq m \leq t \leq \infty]$, we have

$$
\begin{aligned}
& \left|F\left(t, u, x_{1}\right)-F\left(t, u, x_{2}\right)\right| \leqslant \frac{2 m_{3}}{m_{1}-m_{2}}\left|e^{m_{1} t} e^{-\left(m_{1}-2 \alpha\right) u}-e^{m_{2} t} e^{-\left(m_{2}-2 \alpha\right) u}\right| \quad\left|x_{1}-x_{2}\right| \\
& \left|F^{(1)}\left(t, u, x_{1}\right)-F^{(1)}\left(t, u, x_{2}\right)\right| \\
& \quad \leqslant \frac{2 m_{3}}{m_{1}-m_{2}}\left|m_{1} e^{m_{1} t} e^{-\left(m_{1}-2 \alpha\right) u}-e^{+m_{2} t} e^{-\left(m_{2}-2 \alpha\right) u}\right| \quad\left|x_{1}-x_{2}\right|
\end{aligned}
$$


Therefore:

$$
\begin{aligned}
& \left.K_{0}(t, u)=\frac{2 m_{3}}{m_{1}-m_{2}} \mid e^{m_{1} t} e^{-\left(m_{1}-2 \alpha\right) u}-e^{m_{2} t} e^{-\left(m_{2}-2 \alpha\right) u}\right] \\
& K_{1}(t, u)=\frac{2 m_{3}}{m_{1}-m_{2}}\left[m_{1} e^{m_{1} t} e^{-\left(m_{1}-2 \alpha\right) u}-m_{2} e^{m_{2} t} e^{-\left(m_{2}-2 \alpha\right) u}\right] .
\end{aligned}
$$

Applying Equation 8 gives:

$$
n_{0}(t)=\int_{0}^{t} \frac{m_{3}}{\left(m_{1}-m_{2}\right)^{3}}\left[e^{m_{1}(t-u)}-e^{m_{2}(t-u)}\right]\left[-m_{2} e^{m_{1} u}+m_{1} e^{m_{2} u}\right]^{2} e^{2 \alpha u} \mathrm{~d} u
$$

and

$$
n_{1}(t)=\int_{0}^{t} \frac{m_{3}}{\left(m_{1}-m_{2}\right)^{3}}\left[m_{1} e^{m_{1}(t-u)}-m_{2} e^{m_{2}(t-u)}\right]\left[-m_{2} e^{m_{1} u}+m_{1} e^{m_{2} u}\right]^{2} e^{2 a u} \mathrm{~d} u
$$

An evaluation of the integrals of Equations 59 and 60 gives:

$$
\begin{aligned}
& n_{0}(t)=c_{01} e^{2\left(m_{1}+\alpha\right) t}+c_{02} e^{2\left(m_{2}+2 \alpha\right) t}+c_{03} e^{\left(m_{1}+m_{2}+2 \alpha\right) t}+c_{04} e^{m_{1} t}+c_{05} e^{m_{2} t} \\
& n_{1}(t)=c_{11} e^{2\left(m_{1}+\alpha\right) t}+c_{12} e^{2\left(m_{2}+\alpha\right) t}+c_{13} e^{\left(m_{1}+m_{2}+2 \alpha\right) t}+c_{14} e^{m_{1} t}+c_{15} e^{m_{2} t}
\end{aligned}
$$

where

$$
\begin{aligned}
& c_{01}=\frac{m_{3}}{\left(m_{1}-m_{2}\right)^{3}}\left[\frac{m_{2}^{2}}{m_{1}+2 \alpha}-\frac{m_{2}^{2}}{2 m_{1}+2 \alpha-m_{2}}\right] \\
& c_{02}=\frac{m_{3}}{\left(m_{1}-m_{2}\right)^{3}}\left[\frac{m_{1}}{2 m_{2}+2 \alpha-m_{1}}-\frac{m_{1}^{2}}{m_{2}+2 \alpha}\right] \\
& c_{03}=\frac{m_{3}}{\left(m_{1}-m_{2}\right)^{3}}\left[\frac{-2 m_{1} m_{2}}{m_{2}+2 \alpha}+\frac{2 m_{1} m_{2}}{m_{1}+2 \alpha}\right] \\
& c_{04}=\frac{m_{3}}{\left(m_{1}-m_{2}\right)^{3}}\left[\frac{-m_{2}^{2}}{m_{1}+2 \alpha}+\frac{2 m_{1} m_{2}}{m_{2}+2 \alpha}+\frac{-m_{1}^{2}}{2 m_{2}+2 \alpha-m_{1}}\right] \\
& c_{05}=\frac{m_{3}}{\left(m_{1}-m_{2}\right)^{3}}\left[\frac{m_{2}^{2}}{m_{1}+2 \alpha-m_{2}}-\frac{2 m_{1} m_{2}}{m_{1}+2 \alpha}+\frac{m_{1}^{2}}{m_{2}+2 \alpha}\right] \\
& c_{11}=\frac{m_{3}}{\left(m_{1}-m_{2}\right)^{3}}\left[\frac{m_{2}^{2} m_{1}}{m_{1}+2 \alpha}-\frac{m_{2}^{3}}{2 m_{1}+2 \alpha-m_{2}}\right] \\
& c_{12}=\frac{m_{3}}{\left(m_{1}-m_{2}\right)^{3}}\left[\frac{m_{2}^{2} m_{1}}{m_{1}+2 \alpha}-\frac{m_{2}^{3}}{2 m_{1}+2 \alpha-m_{2}}\right] \\
& c_{13}=\frac{m_{3}}{\left(m_{1}-m_{2}\right)^{3}}\left[\frac{-2 m_{1}^{2} m_{2}}{m_{2}+2 \alpha}+\frac{2 m_{1} m_{2}^{2}}{m_{1}+2 \alpha}\right] \\
& c_{14}=m_{1} c_{04} \\
& c_{15}=m_{2} c_{05}
\end{aligned}
$$


Applying the conditions of Equation 8 gives:

$$
\begin{aligned}
& \alpha_{0}^{2}(t)=\int_{0}^{t} K_{0}^{2}(t, u) \mathrm{d} u=d_{01} e^{\left(m_{1}+m_{2}\right) t}+d_{02} e^{2 m_{1} t}+d_{03} e^{2 m_{2} t}+d_{04} e^{4 \alpha t} \\
& \alpha_{1}^{2}(t)=d_{11} e^{\left(m_{1}+m_{2}\right) t}+d_{12} e^{2 m_{2} t}+d_{13} e^{2 m_{2} t}+d_{14} e^{-4 \alpha t}
\end{aligned}
$$

where

$$
\left.\begin{array}{l}
d_{01}=\frac{4 m_{3}^{2}}{\left(m_{1}-m_{2}\right)^{2}}\left[\frac{2}{m_{1}+m_{2}-4 \alpha}\right] \\
d_{02}=\frac{4 m_{2}^{2}}{\left(m_{1}-m_{2}\right)^{2}}\left[\frac{1}{2\left(m_{1}-2 \alpha\right)}\right] \\
d_{03}=\frac{4 m_{3}^{2}}{\left(m_{1}-m_{2}\right)^{2}}\left[\frac{1}{2\left(m_{2}-2 \alpha\right)}\right] \\
d_{04}=\frac{4 m_{3}^{2}}{\left(m_{1}-m_{2}\right)^{2}}\left[\frac{1}{2\left(m_{1}-2 \alpha\right)}+\frac{1}{2\left(m_{2}-2 \alpha\right)}-\frac{2}{\left(m_{1}+m_{2}-4 \alpha\right)}\right] \\
d_{11}=m_{1} m_{2} d_{01} \\
d_{12}=m_{1}^{2} d_{02} \\
d_{13}=m_{2}^{2} d_{03} \\
d_{14}=\frac{4 m_{3}^{2}}{\left(m_{1}-m_{2}\right)^{2}}\left[\frac{m_{1}^{2}}{2\left(m_{1}-2 \alpha\right)}+\frac{m_{2}^{2}}{2\left(m_{2}-2 \alpha\right)}-\frac{2 m_{1} m_{2}}{m_{1}+m_{2}-6 \alpha}\right]
\end{array}\right\}
$$

The functions $x_{0}^{(i)}(t), n_{i}(t)$, and $\alpha_{i}(t)$ for $i=0$ and 1 can be easily shown to belong to an $L_{2}$ space in $0 \leq t \leq \infty$, if the values of $m_{1}, m_{2}$, and $\alpha$ (the system parameters) are properly chosen. These functions are also uniformly continuous and therefore will have zero limits at $t=+\infty$.

With these results and utilizing the theory presented, we can draw the following conclusions:

(1) The system has a unique response in $0 \leq t \leq \infty$.

(2) The actuating signal, $v_{1}(t)$, and the rate of change of this signal $\dot{v}_{1}$, belong to an $L_{2}$ space in $0 \leq t \leq \infty$; that is, both the integrals

exist and are less than a given number.

$$
\int_{0}^{\infty} v_{1}^{2}(t) \mathrm{d} t \text { and } \int_{0}^{\infty} \dot{v}_{1}^{2}(t) \mathrm{d} t
$$

(3) $\operatorname{Lim}_{t \rightarrow \infty} v_{1}(t)=\operatorname{Lim}_{t \rightarrow \infty} \dot{v}_{1}(t)=0$.

In order to evaluate the two integrals in item (2) above, we must use Equations 29 and 30 , from which it is necessary to evaluate the two constants $A_{0}$ and $C_{0}$ as follows:

$$
\begin{aligned}
& A_{0}=\left[\int_{0}^{\infty} \alpha_{0}^{2}(t) \mathrm{d} t\right]^{\xi}=\left(\frac{-d_{01}}{m_{1}+m_{2}}-\frac{d_{02}}{2 m_{1}}-\frac{d_{03}}{2 m_{2}}-\frac{d_{04}}{2 \alpha}\right)^{\frac{1}{2}} \\
& C_{0}=\left[\int_{0}^{\infty} n_{0}^{2}(t) \mathrm{d} t\right]^{\frac{1}{2}} \leqslant 4\left(\frac{-c_{01}{ }^{2}}{4\left(m_{1}+\alpha\right)}-\frac{c_{02}{ }^{2}}{4\left(m_{2}+\alpha\right)}-\frac{c_{03}{ }^{2}}{2\left(m_{1}+m_{2}+2 \alpha\right)}-\frac{c_{04}{ }^{2}}{m_{1}}-\frac{c_{05}{ }^{2}}{m_{2}}\right)^{\frac{1}{2}} .
\end{aligned}
$$
inequality $(a+b+c+d+e)^{2} \leqslant 4\left(a^{2}+b^{2}+c^{2}+2 d^{2}+2 e^{2}\right)$. 
Furthermore, evaluation of the integrals in Equations 67 and 68 show that the requirement given by Equation 48 can be satisfied by choosing the following set of parameters:

$$
\left.\begin{array}{l}
J_{a}=K_{a}=K_{b}=1 \\
B=K_{c}=2 \\
\alpha=-1.5
\end{array}\right\}
$$

which corresponds to $m_{1}=-1, m_{2}=-2, m_{3}=1$, and $\alpha=-1 \cdot 5$, and (see Equation 46)

$$
\begin{aligned}
& K_{12}=-1 \\
& K_{22}=-3 \\
& K_{21}=+2
\end{aligned}
$$

\section{CONCLUSIONS AND REMARKS}

The partitioning technique and the state variable approach have been applied to study the behaviour of a nonlinear physical system whose dynamic performance can be represented by a nonlinear differential equation containing some linear terms, some nonlinear terms, and a forcing function term as defined by Equation 1. It was found [1] that the response function $x(t)$ of the system defined by Equation 1 possesses a unique solution which is the limit of a sequence of iterates in the $L_{2}$ space. As shown here, under suitable restrictions, this solution as well as the state variables, $v_{1}, v_{2} \ldots, v_{n}$, belong to an $L_{2}$ space. The system as restricted was found to satisfy LYAPUNOV's definition of asymptotic stability. Hence the restrictions placed on the system are sufficient not only to prove the uniqueness of a solution that belongs to an $L_{2}$ space but also to produce, in the sense indicated by LYAPUNOV, asymptotically stable systems. The behaviour of the system trajectory at any time $t$ and during the average interval of interest has been examined with respect to two different definitions of the norm.



FIO. 3. The actual and average system responses.

In many applications, studying system trajectory averages is usually unsatisfactory, since they may not exceed certain specified values, whereas an actual system's response may assume an undesirable shape when plotted, as Fig. 3 shows. For this reason, an expression for an upper-bound state vector inside which the system will always remain during operation has been derived. The upper-bound state vector is found to depend only upon the restrictions placed on the system, and hence can be varied to suit specific applications by changes in the system parameters. An example to illustrate the method is given. 
The authors believe that the analysis presented herein should assist engineers in analyzing or synthesizing systems containing nonlinear characteristics.

\section{APPENDIX A}

To prove that:

$$
\operatorname{Lim}_{m \rightarrow \infty} x_{m}^{(k)} \rightarrow x^{(k)}=v_{k+1}
$$

where $x_{m}^{(k)}$ and $x^{(k)}$ are given by Equations 22 and 20, respectively:

$$
\begin{aligned}
& x^{(k)}=v_{k+1}=x_{0}^{(k)}(t)-\int_{0}^{t} F^{(k)}\left[t, u, v_{1}(u)\right] \mathrm{d} u \\
& x_{m}^{(k)}=x_{0}^{(k)} t-\int_{0}^{t} F^{(k)}\left[t, u, v_{m-1}(u)\right] \mathrm{d} u .
\end{aligned}
$$

The proof should be divided into two steps:

(1) To prove that $\operatorname{Lim} x_{m}^{(k)}$ exists.

(2) To prove that the limit of step 1 satisfies Equation 27.

For step 1, we can form the series

$$
x_{m}^{(k)}=x_{1}^{(k)}+\left[x_{2}^{(k)}-x_{1}^{(k)}\right]+\left[x_{3}^{(k)}-x_{2}^{(k)}\right]+\ldots \text { to } m+\text { errors } .
$$

Therefore:

$$
x_{m}^{(k)} \leqslant x_{1}^{(k)}+\alpha_{k}(t) C_{0}+C_{0} \alpha_{k}(t) \sum_{m=2}^{\infty} \frac{A_{0}^{m-1}}{\sqrt{(m-2) !}} .
$$

Equation 71 shows that, as $m \rightarrow \infty, x_{m}^{(k)}$ has a limit, which proves step 1 .

For step 2, subtracting Equation 20 and 22 and squaring gives

$$
\begin{aligned}
& {\left[x_{m}^{(k)}-v_{k+1}\right]^{2}=\left[\int_{0}^{t}\left[F^{(k)}\left(t, u, v_{m-1} u\right)-F^{(k)}\left(t, u, v_{1}(u)\right] \mathrm{d} u\right]^{2}\right.} \\
& {\left[x_{m}^{(k)}-v_{k+1}\right]^{2}=\left[\int_{0}^{t} K_{k}(t, u)\left|v_{m-1}(u)-v_{1}(u)\right| \mathrm{d} u\right]^{2} .}
\end{aligned}
$$

Therefore

$$
\left[x_{m}^{(k)}-v_{k+1}\right]^{2} \alpha_{k}^{2}(t) \int_{0}^{T}\left|v_{m-1}(u)-v_{1}(u)\right| \mathrm{d} u .
$$

It is proved in Ref. 4, Appendix B, that

$$
\operatorname{Lim}_{m \rightarrow \infty} v_{m-1}(u)=v_{1}(u)
$$


Hence:

$$
\operatorname{Lim}_{m \rightarrow \infty} x_{m}^{(k)}=v_{k+1}
$$

It should not be necessary to prove that, as $m \rightarrow \infty, x_{m}^{(k)} \rightarrow v_{k+1}$ uniquely, since it was proved in Ref. 4, Appendix B, that, as $m \rightarrow \infty, v_{m-1}(u)$ tends toward $v_{1}(u)$ uniquely.

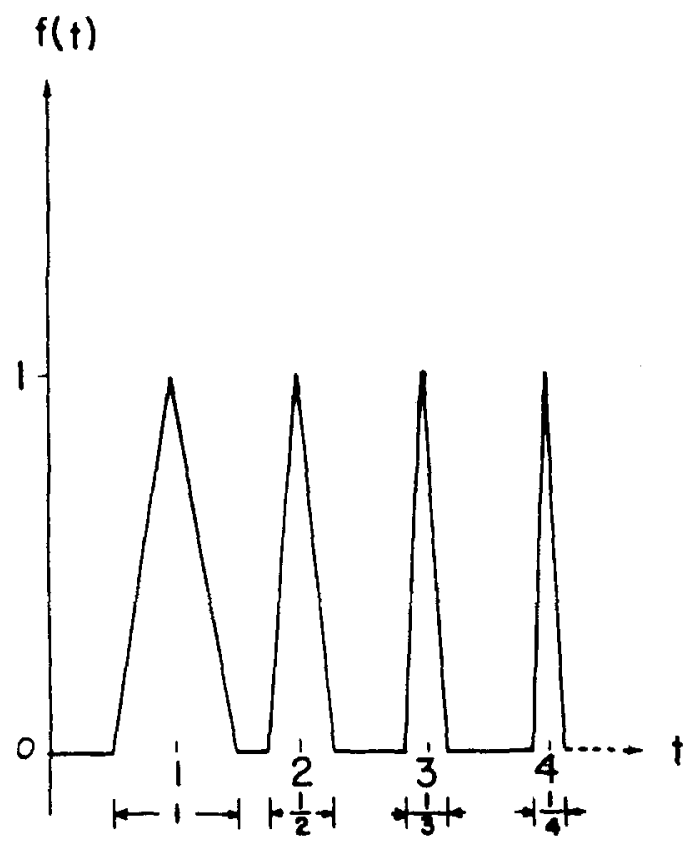

FIG. 4. A function which belongs to $L_{2}$, but the limit at $t=\infty$ does not exist.

\section{APPENDIX B}

An example follows in which the function is $L_{2}$ on $[0+\infty)$, but the limit does not exist. Consider the function shown in Fig. 4, where

$$
\int_{0}^{\infty} f^{2}(t) \mathrm{d} t=\frac{1}{4} \sum_{n=1}^{\infty} \frac{1}{n^{2}} .
$$

The infinite series of Equation 75 converges, and therefore the integral exists, but the value of the function $f(t)$ at $t=+\infty$ does not exist.

Acknowledgment-The authors wish to acknowledge the assistance of the Engineering Faculty, in particular to Dr. W. A. Porter of the Navigation and Guidance Laboratory of the Institute of Science and Technology.

Résumé-La technique de partition et l'approche de la variable d'état ont été appliquées à l'analyse et à l'étude du comportement dynamique d'un système de commande dont la performance dynamique peut être genèralement décrite à l'aide d'une équation differentielle non-lineaire contenant quelques termes linéaires, quelques termes non-linéaires et un terme de fonction d'intensification. En appliquant à l'équation du système des limitations convenables, il a été trouvé que les variables d'état, qui reprèsentent l'état du système appartiennent à un espace. Sous la reserve des limitations adoptées il a été trouvé que le système admissible satisfaisait a la définition de la stabilité asymptotique dans le sens de 
Liapunov. La trajectoire du système a été analyse en utilisant deux définitions différentes de la norme. On a obtenu, pendant l'intervalle de fonctionnement considéré des expressions pour les normes nécessaires à l'intérieur desquelles se trouvent tous les états possibles du systéme. On trouvera dans l'article un exemple illustrant la méthode présentée.

Zusammenfassung-Die "partitioning"-Technik und die Methode der Zustands-Variablen wurde angewandt, um das Verhalten eines Regelungssystems zu alanysieren und zu studieren, dessen dynamisches Verhalten im allgemeinen durch eine nichtlineare Differentialgleichung beschrieben werden kann, wobei es sowohl lineare als auch nichtlineare und schliesslich Terme von Störfunktionen enthalten kann. Unter Einführung geeigneter Voraussetzungen bei der Systemgleichung wurde gefunden, dass die Variablen, die den Zustand des Systems repräsentieren, einem $\mathrm{L}_{2}$-Raum angehören. Bei den getroffenen Voraussetzungen befriedigt das zulässige System die Definition der asymptotischen Stabilität im Sinne von Ljapunow. Unter Benutzung zweier verschiedener Definitionen der Norm wurde die Bahnkurve des Systems analysiert. Für das betrachtete Intervall wurden Ausdrücke für die geforderten Normen erhalten, innerhalb welcher alle möglichen Zustände des Systems bleiben. Um die vorliegende Methode zu illustrieren, wurde ein Beispiel angeschlossen.

Абстракт-Техника разделения и подход переменной состояния ъыли применны к анализу и к изучению динамического поведения системы управления, динамические характеристики которой, могут ъыть, в далышинстве случаев, описаны при помощи нелинейного диференциального уравнения содержашего несколько линейных членов, несколько нелинейньх членов и член вынужцающей функция. Применяя к уравнению системы подходящие ограничения, ъыло показаночто, переменные состояния описываюшие состояние системы, принадлежат к некоторому пространству $Л_{2}$. Ыыло показано что, при принятых огранчентях, приемлемая система удовлетвояла определению асимптоматической устойчивости в смысле Лягунова. Траектория системы ъыла проанализирована при помощи двух различных опрелений нормы, ыыли получены, для рассматриваемого интервала действия, выражения для неоъходимых норм, внутри которых находятся все возможные состояния системы. Статья содержит пример иллюстрирующий представленный метод. 